\title{
POSTPRANDIAL HYPOGLYCAEMIA IN NON-DIABETICS MAY REVEAL 눈 PATIENTS WITH DIABETES-RELATED ANTIBODIES, AND AT DIABETES RISK
}

\section{S. Ljubic ${ }^{1,2}$, A. Jazbec ${ }^{2}$, M. Vucic Lovrencic ${ }^{1}$, M. Tomic ${ }^{1}$ A. Piljac ${ }^{1}$, L. Smircic-Duvnjak ${ }^{1,2}$ Merkur Clinical Hospital, Vuk Vrhovac University Clinic ${ }^{1}$, University of Zagreb ${ }^{2}$, Zagreb, Croatia}

Our observation was that some non-diabetic patients who manifest postprandial hypoglycaemia (PPH) may develop glucose intolerance (GI) or diabetes mellitus (DM). The study was performed to determine relation between PPH and GI or DM.

\section{PATIENTS AND METHODS}

The duration of the study was over a 10 year interval. Patients were tested on the presence of insulin receptor (IRAb), islet cell (ICAs), glutamic acid decarboxylase (GAD) and antithyroid Abs (TAbs) antibodies.

Prolonged (4h) OGTT was performed in 216 tested patients. Blood glucose (BG), C-peptide (CP) and insulin levels, insulin resistance (IR), C-reactive protein (CRP), homocysteine, liver function tests, lipids and other metabolic syndrome (MS) parameters, as well as cardiovascular events (CVE) and DM in relatives were also determined.

Patients were allocated in the groups according to the presence of Ab. Subgroups are defined according to the development of GI or DM in Group 0 and Group 1.

\begin{tabular}{|c|c|c|c|}
\hline \multicolumn{2}{|c|}{ Group 0 } & \multicolumn{2}{c|}{ Group 1 } \\
\hline \multicolumn{2}{|c|}{ ICA/GAD neg. } & \multicolumn{2}{|c|}{ ICA and/or GAD pos. } \\
\hline Subgroup 3 & Subgroup 4 & Subgroup 5 & Subgroup 6 \\
\hline No GI/DM & With GI/DM & No GI/DM & With GI/DM \\
\hline
\end{tabular}

RESULTS

Prolonged (4h) OGTT demonstrated marked hypoglycaemia in $180 \mathrm{~min}$. Positive IRAb have been presented in only 1 patient.

ICA and/or GAD were positive in $30.41 \%$ patients with $\mathrm{PPH}$.

- Significantly higher values (Student's $t$ test) were determined in Group 0 in comparison with group 1 in fasting BG (FBG), fasting insulin (FINS), IR and CRP.

\begin{tabular}{|l|c|c|c|}
\hline \multicolumn{5}{|c|}{ Comparison between tested variables between } \\
\multicolumn{4}{|c|}{ Group 0 and Group 1 } \\
\hline FBG (mmol/L) & $4.86 \pm 0.73$ & Group 1 & p-value \\
\hline FINS $($ pmol/L) & $60.27 \pm 35.97$ & $48.56 \pm 0.73$ & 0.02 \\
\hline IR & $1.59 \pm 1.01$ & $1.17 \pm 0.96$ & 0.04 \\
\hline CRP $(m g / L)$ & $1.48 \pm 1.2$ & $1.01 \pm 0.62$ & 0.01 \\
\hline
\end{tabular}

Two groups also differ in the presence of TAbs: TAbs were positive in $34.38 \%$ of Group 1 patients and in $14.75 \%$ of Group 0 patients.

ANOVA revealed significant difference $(p<0.05)$ in glycated haemoglobin $(\mathrm{HbA} 1 \mathrm{c})$, postprandial CP (PCP), IR, CRP, systolic blood pressure (SBP), aspartate transaminase (AST) $(p=0.05)$, alanine transaminase (ALT), gamma-glutamyl transpeptidase (GGT) and triglycerides (TG) among subgroups, with no difference in body mass index.

There was no difference in CVE and DM in relatives between subgroups.

\begin{tabular}{|l|c|c|c|c|}
\hline & \multicolumn{2}{|c|}{ CVE in relatives } & \multicolumn{2}{c|}{ DM in relatives } \\
\hline & Group 0 & Group 1 & Group 0 & Group 1 \\
\hline Subgroup & \multicolumn{4}{|c|}{ Number of patients } \\
\hline 3 & 47 & 24 & 29 & 42 \\
\hline 4 & 13 & 7 & 7 & 13 \\
\hline 5 & 13 & 13 & 11 & 15 \\
\hline 6 & 7 & 5 & 6 & 6 \\
\hline
\end{tabular}

\section{CONCLUSIONS}

High percentage of ICAs and/or GAD positive Ab accompanied PPH in non-diabetic patients and could be the reason for GI and DM development, whereas MS parameters might be relevant in ICAs and GAD-Ab negative subjects.

PPH in non-diabetic patients may reveal patients with an increased risk of DM; if ICAs and/or GAD-Ab are positive latent autoimmune diabetes of adults could be considered.
Patients in subgroup 4 (15.75\% of tested patients) had significantly higher values of HbA1c, IR, CRP, ALT, GGT and TG in comparison with other tested subgroups, whereas AST and PCP were significantly higher in subgroup $6(9.45 \%$ of tested patients).

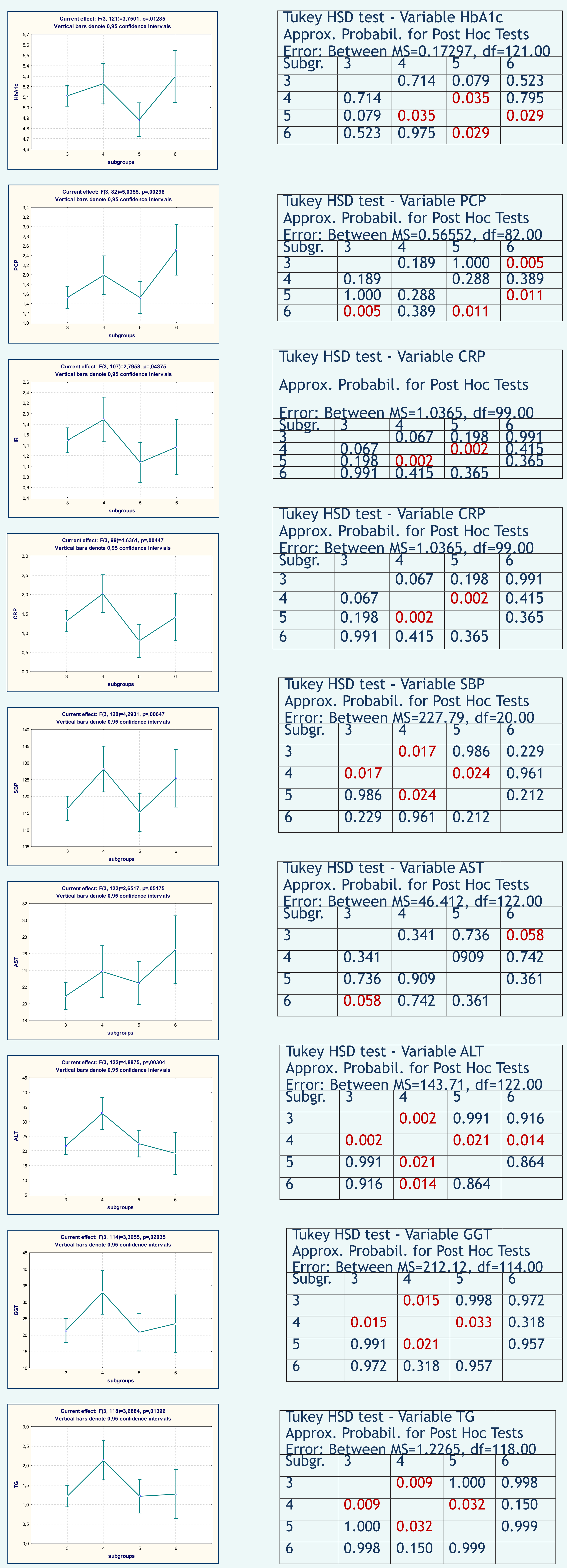

